\title{
SYMPOSIA ORAL PRESENTATIONS
}





\title{
Systematics, Taxonomy, Distribution, Ecology, and Fungal Culture Collections
}

\section{Molecular Diversity of Natural Tuber Species from Slovenia}

\author{
Tine Grebenc, ${ }^{1}$ Maria P. Martin, ${ }^{2}$ Andrej Piltaver,, 3 Ivan Ratoša, 4 and Hojka Kraigher ${ }^{1}$
}

${ }^{1}$ Slovenian Forestry Institute, Večna pot 2, Ljubljana SI-1000, Slovenia; ${ }^{2}$ Real Jardin Botanico, Plaza de Murillo 2 , Madrid 28014, Spain; ${ }^{3}$ Institute for Systematics of Higher Fungi, 24 Z. Kvedrove, Ljubljana, Slovenia; ${ }^{4}$ Ivan Ratoša, Goriče 3, Postojna 6230, Slovenia

Mycorrhizal fungi, belonging to the genus Tuber, produce hypogeous fruit bodies, some of them of great economic value known as truffles. Currently, the genus Tuber comprises about 63 valid species, some of which possess high economic value and their identification and separation from lower quality species is crucial. Slovenia is not yet well known as a country of truffles culture. Implementation of suitable analysis tools, molecular markers, and reference material is necessary for proper identification of the collections and assessment of putative diversity of collections. Therefore, the collected material was analyzed for diversity of rDNA ITS spacers and $\beta$-tubulin regions, and compared to the reference herbarium material.

We have analyzed about 150 samples collected in 2004 and 2005, mainly belonging to the genus Tuber and some other hypogeous sporocarps, collected together with truffles within the truffle grounds, which were analyzed for identification only. Reference sporocarps were obtained from the herbarium at the Real Jardín Botánico, Madrid (collection MA-Fungi). The material was analyzed for diversity of rDNA ITS spacers and $\beta$-tubulin regions.

About $20 \mathrm{mg}$ of the hymenium from the inner part of ascocarps was used for the DNA extraction following standard protocols after Whiting et al. (Syst Biol, 1999, 46:1-68). Primers ITS1 $\mathrm{f}^{5}$ or ITS5 $5^{5}$ and ITS4 ${ }^{4}$ were used for PCR amplification and sequencing of the ITS region, including 5.8S rDNA. The PCR reactions were performed after Kraigher et al. with an annealing temperature of $55^{\circ} \mathrm{C}$ (Kraigher et al., Mycorrhiza, 1995, 5:175-180). DNA was sequenced in both directions using the ITS $1 \mathrm{f}$ or ITS5 and ITS4 primers. Obtained sequences were arranged with the Sequencher 4.6 Sequence Analysis software (Gene Code Corporation) and compared to the GenBank database (http://www.ncbi.nlm.nih.gov/) for the identification. Different phylogenetic analyses were performed for genus Tuber using the program PAUP *Version 4.0b10 for Macintosh.

Comparing existing and obtained sequences of the ITS region in nuclear RNA genes, we managed to separate and identify most of the commercially important species from the material collected from Slovenia and the neighboring areas, comparing them to material from herbarium RJB and available sequences in GenBank. Molecular data confirmed results of the recent 2-year inventory that proved the presence of all commercially important truffle species including highly precious $T u$ ber magnatum Pico and T. melanosporum Witt. in Slovenia. Amplified DNA sequences of hypogeous fungi subjected to phylogenetic relationship analysis showed clear separation of black-spore and whitespore species. Most of the analyzed species did not show any intraspecific polymorphism in the ITS regions. An exception was T. rufum Pico, which showed clear variability of the analyzed DNA region between different geographic regions. Further sampling of $T$. rufum is necessary for more detailed analysis of the observed polymorphism.

Primers for amplification of $\beta$-tubulin regions constructed for Basidiomycetes showed only limited use for truffles. One part of the region was successfully amplified using primers B43\&B41, yielding a fragment of about $500 \mathrm{bp}$. Amplified introns showed some variability but lower than the rDNA ITS regions. Only a few sited introns in the amplified $\beta$-tubulin region were informative. The $\beta$-tubulin regions were not used for further phylogenetic analysis.

\section{ACKNOWLEDGMENTS}

Results were conducted as a part of SYNTHESYS ES-TAF-1729 - financial support of the European 
Community's Program "Structuring the European Research Area", under SYNTHESYS at the Real Jardín Botánico de Madrid (CSIC). The partners from the Slovenian Forestry Institute were co-financed by the Ministry for Higher Education, Science, and Technology through the Research Program P4-0107, project L1-6706 (co-financed by the Ministry for Agriculture, Forestry, and Food).

\title{
Innovation Systems and Cluster Approaches to Exploitation of the Indigenous and Exotic Edible and Medicinal Mushrooms of the Lake Victoria Basin
}

\author{
Deogracious Olila, John D. Kabasa, Leonard Kisovi, Andrew Engola, \\ and Pataleo K. Munishi \\ Department of Veterinary Physiological Sciences, Makerere University, P.O. Box 7062, Kampala, Uganda
}

Mushrooms are used as food and medicine in many societies. They are also of considerable biotechnological value and important as nutraceuticals, particularly in immuno-compromised patients (e.g., HIV cases). However, the nutritional, ecological, medicinal, and biotechnological importance of indigenous Ugandan mushrooms are not adequately studied and documented.

Faced with a poor and rapidly growing population in Uganda, the competition for natural resources by different producers is increasing. Expansion of crop and animal production has adverse effects on the environment. There is increasing pressure on land, leading to loss of indigenous mushroom biodiversity without understanding their contribution to present and future generations.

This paper will present some data on the characterization of indigenous mushroom ecology, their cultivation, indigenous knowledge, domestication and assessment of their potential as sources of nutrients, novel antibiotics, and metabolites for improved health and socio-economic benefits. The informa- tion was gathered through reconnaissance surveys, participatory appraisals, and standard laboratory techniques involving assessment of nutritional and pharmacological potential.

Innovation systems and cluster approaches are presented as a way to maximize exploitation of the indigenous African mushroom biodiversity. This is because, today, economic development policies that target individual firms or industries are no longer the most viable options for regions. Cluster development has gained considerable attention over the past decade, as an operational means to systematically enhance the competitiveness of regions. The new economy of clusters has to do with speed, quality, flexibility, innovation, networks, and building critical mass. This new style demands a team approach at the local level, a clustering approach. This cluster approach is the missing link in the indigenous mushroom industry in Africa. It is only through developing viable clustering that even small firms are able to gain the necessary critical mass to service world markets. 


\title{
Gene Bank of the Cultivatable Higher Basidiomycetes Species in the Hungarian Mushroom Industry
}

\author{
József Szarvas, ${ }^{1}$ Laszló Bujdosó, ${ }^{1}$ Csaba Hajdú, ${ }^{1,2}$ and Melinda Bray ${ }^{1}$ \\ ${ }^{1}$ National Korona Mushroom Union, Strain Research and Molecular Biological Laboratory, P.O. Box 1., Demjén \\ H-3395, Hungary; ${ }^{2}$ Szent István University, School of Horticultural, Ménesi út 44., Budapest H-1118, Hungary
}

Recently, the protection of living creatures' genome gets more and more significance. The increasing environmental harm reduces the chances for some populations to survive. Therefore, it is necessary to protect the endangered mushroom species and their habitats. In the case of endangered higher Basidiomycetes, considering the availability of fruiting bodies, we can assume that a population exists. However, the lack of fruiting bodies is not always connected with populational loss.

Besides the protection of mushroom species and their habitats, the most effective solution is the preservation of the species in the form of laboratory cultures. Our laboratory has undertaken to establish the stock breeding mainly of edible mushrooms. In Hungary, it is the only place keeping not only numerous wild species but also a collection of hybrids and basic material used for breeding from 20th century-breeders, such as the famous oyster mushroom hybrids (HK-35, HK-44, H-7, etc.) of Pál Gyurkó. The importance of these, as our national values is huge, furthermore they supply excellent basic material for breeding.

In order to keep the stability of our genetic cultures we applied cryopreservation techniques in the case of some mushroom species, stocks, and hybrids. We determined the cryoprotective materials, the method of freezing and melting, and exact database for registration the place of certain cultures. The refilling of the cryopreservator continues.

\section{Taxonomy and Molecular Phylogeny within the Pleurotus eryngii Species-Complex}

\author{
Georgios I. Zervakis, ${ }^{1}$ Giuseppe Venturella, ${ }^{2}$ Maria Bessi, ${ }^{1}$ Maria Letizia Gargano, ${ }^{2}$ \\ Zhi-Hui Yang, ${ }^{3}$ and Yi-Jian Yao ${ }^{3}$ \\ ${ }^{1}$ National Agricultural Research Foundation, Institute of Kalamata, Kalamata, Greece; ${ }^{2}$ University of Palermo, \\ Department of Botany, Via Archirafi 38, Palermo, Italy; ${ }^{3}$ Chinese Academy of Sciences, Institute of Microbiology, \\ Beijing 100101, China
}

The Pleurotus eryngii species-complex comprises edible fungi with a large commercial and biotechnological potential, which is characterized by the development of weak (facultative) biotrophic associations with host plants of the families Apiaceae and Compositae. However, the taxonomic relationships within this group are ambiguous, and this is due to initial misidentifications, erroneous application of taxa names, absence of species typification, and the influence exercised by the environment on the phenotype of each taxon.

Recent studies based on isozyme and RAPD-PCR analysis showed that Pleurotus isolates from Europe growing on plants of the genus Cachrys formed a distinct cluster with a relatively high statistical support presenting low intra-population variability, and increased genetic distances with populations from other associated plants. In contrast, $P$. eryngii strains isolated from Eryngium, Ferula, Elaeoselinum, and Thapsia were positioned within a single large cluster, which was further subdivided into four main groups corresponding to each one of those hosts.

In the framework of this work, 40 representative strains of the Pleurotus eryngii species-complex isolated from a large range of host plants (e.g., Eryngium campestre, E. maritimum, E. amethystinum, Ferula 
communis, F. tingitana, F. sinkiangensis, Ferulago galbanifera, Cachrys ferulacea, Laserpitium latifolium, Elaeoselinum asclepium ssp. asclepium, E. gummiferum, Thapsia garganica, T. villosa, etc.), with a wide geographical distribution (Mediterranean Europe, Middle East, Asia), were studied by using ecomorphological characteristics and molecular techniques, i.e., sequencing of: the internally transcribed spacers (ITS 1 and ITS 2); the intergenic spacers (IGS), and; the 5.8S rDNA gene. In addition, biological material originating from the $\mathrm{M}$, GDOR, PAL, PC, FI, GE, UPS, BERN, K, PI, and IB Herbaria was examined and evaluated.

The results demonstrated the existence of two distinct species, $P$. eryngii and $P$. nebrodensis. The former included several established varieties, on one hand, all primarily based on the identity of the host plant (Eryngium, Ferula, Thapsia, Elaeoselinum, and Laserpitium) and on their morphology and type of habit/habitat. On the other hand, $P$. nebrodensis was found in confined populations with a rather limited number of individuals, and in association with the plant Cachrys ferulacea. Furthermore, populations growing in Asia on Ferula sinkiangensis and in Europe on Ferulago galbanifera seemed to be rather separated from the two well-established species of the complex. Hence, host-specificity appears to play a key role in speciation processes within this particular group of oyster mushrooms, as opposed to what is the case for most other Pleurotus species, where geographic origin is the determinant factor.

\section{ACKNOWLEDGMENTS}

This work was funded by the Joint Research and Technology Programmes between Greece and China, and by the EPAN-FP66 research project (both supported by the Greek General Secretariat of Research and Technology).

\title{
Classification of Ganoderma Strains Cultivated in China Using Molecular Methods
}

\author{
Jingsong Zhang, ${ }^{1}$ Chuanhong Tang, ${ }^{1}$ Chunli Su, ${ }^{1,2}$ Mingjie Chen, ${ }^{1}$ Hui Cao, ${ }^{1}$ \\ Qi Tan, ${ }^{1}$ and Yingjie Pan ${ }^{3}$ \\ ${ }^{1}$ Institute of Edible Fungi, The Shanghai Academy of Agricultural Sciences, Shanghai 201106, China; ${ }^{2}$ Microbiology \\ Department, Nanjing Agriculture University, Nanjing 201195, China; ${ }^{3}$ College of Food Science, Shanghai Fisheries \\ University, Shanghai 200090, China
}

In order to classify strains of Ganoderma cultivated in China, genetic diversity among 150 strains of Ganoderma was studied using RAPD (Random Amplified Polymorphic DNA) and ERIC-PCR (Enterobacteria Repetitive Intergenic Consensus-PCR) techniques and inferred from nuclear ribosomal DNA ITS and partial $\beta$-tubulin gene sequences. Two dendrograms, derived from RAPD and ERIC-PCR data respectively, indicated that the strains could be divided into four distinct groups, and the results obtained were in complete accordance with traditional classification. Compared to the RAPD method, ERIC-PCR is a cheaper and faster method for classifying Ganoderma strains.
Genetic diversity among 34 Ganoderma isolates cultivated in China was investigated using ITS and partial $\beta$-tubulin gene sequences. Five distinct groups were identified. In addition, ITS sequences were used to design primers capable of clearly distinguishing $G$. sinense, G. tsugae, G. tenue, G. subamboinense, and $G$. applanatum using PCR-based technology.

The results emphasize the difficulties involved in identifying Ganoderma isolates using only morphological characteristics, and demonstrate that a more effective approach to resolving the taxomomy of Ganoderma is available through analysis of molecular data. 


\title{
Analysis of Ganoderma Strains Using AFLP and Microscopic Examination of Spores
}

\author{
Linyong Zheng, ${ }_{1,2}$ Dinghong Jia, ${ }^{1}$ Xiaofan Fei, ${ }^{3}$ Xia Luo, ${ }^{1,4}$ Jian Zhao, ${ }^{2,4}$ \\ and Zhirong Yang ${ }^{2}$
}

${ }^{1}$ Soil and Fertilizer Institute, Sichuan Academy of Agricultural Sciences, Chengdu 610066, China; ${ }^{2}$ Key Laboratory of Biological Resource and Ecological Environment of the Ministry of Education, College of Life Sciences, Sichuan University, Chengdu 610064, China; ${ }^{3}$ West China Hospital Sichuan University, Chengdu 610061, China; ${ }^{4}$ Sichuan Institute of Chinese Material Medica, Chengdu 610041, China

Ganoderma lucidum (W. Curt.: Fr.) P. Karst. is one of the most important medicinal materials. The fruit body of G. lucidum, with its perceived health benefits, has gained wide, popular use as a dietary supplement in China, Japan, North America and other regions of the world. As a kind of health food, it also has been used in the prevention and treatment for immunological diseases such as hypertension, tumorigenesis, etc. Furthermore, some Ganoderma species play an important role in plant pathogens in khair (Acacia catechu), grapevines, oil palms, and other economically important tropical crops. Despite its importance, the genetic background, however, is relatively unclear because of its specific interhybridization, with the result that Ganoderma species identification and circumscription were often unclear and a taxonomic segregation of the genus remained controversial. Even a number of Ganoderma isolates have been misnamed. DNA fingerprinting techniques, however, would be allowed to identify the Ganoderma species and cultivars, and also would be a useful tool for the valid protection of newly bred cultivars. A variety of laboratory-based techniques have been used to study genetic diversity in Ganoderma such as isozyme analysis, random amplified polymorphism DNA (RAPD), Amplified Fragment Length Polymorphism Fingerprinting (AFLP) ITS (internal transcribed spacers) and 25S ribosomal DNA sequencing technique, PCR-RFLP. The AFLP technique is based on the PCR amplification of a fraction of restriction fragments generated by the digestion of total DNA, and has merits of RFLP and RAPD. AFLP fingerprinting was used as a tool to study propagation and genetic diversity. The AFLP method has wide applications in molecular biology, genetics, and breeding. In order to study Ganoderma strains' genetic relationship, the AFLP technique was used because of its high resolution.

In this study, AFLP analysis using 14 primer combinations and microscopic examination of spores were carried out on 18 Ganoderma strains to analyze the genetic diversity on a molecular level and Ganoderma spores' morphological differences, respectively.

Results showed that there were 177 polymorphic bands with 14 primer combinations. There was clear fingerprinting with primer pairs of E-ACG/M-CAA and E-ACG/M-CTG, and also revealed that similarity coefficients ranged from 0.07692 to 0.99194 . All accessions could be uniquely identified in AFLP. With UPGMA cluster method, all the strains were clustered into two monophyletic groups: G. lucidum group and G. sinense group. Cluster results supported the morphological taxonomical system in which the subgenus Ganoderma is composed of Sect. Ganoderma and Sect. Phaeonema. Microscopic examination results showed that the spores of $G$. sinense group were brown, and bigger than spores of G. lucidum. Spore surfaces of strains of $G$. sinense group were rugose compared with the spores of G. lucidum group. It had fallen into two obviously different types and inosculated with AFLP clustering results. All strains were clustered into two monophyletic groups with UPGMA method in AFLP and two spore morphological groups in microscopic examination. The results were in accordance with the traditional taxomomic system in which the subgenus Ganoderma is composed of Sect. Ganoderma and Sect. Phaeonema. The congruency of the experiments was analyzed using the biostatistical software NTSYSpc2.1. 